\title{
On existence of the support of a Borel measure
}

https://doi.org/10.1515/dema-2018-0010

Received November 22, 2017; accepted April 12, 2018

Abstract: We present arguments showing that the standard notion of the support of a probabilistic Borel measure is not well defined in every topological space. Our goal is to create a "very inseparable" space and to show the existence of a family of closed sets such that each of them is of full measure, but their intersection is empty. The presented classic construction is credited to Jean Dieudonné and dates back to 1939. We also propose certain, up to our best knowledge, new simplifications.

Keywords: set theory, ordinal numbers, measure

MSC: Primary: 28E15, Secondary: 03E10, 28A05

\section{Introduction}

Let us consider an arbitrary Borel measure defined on a topological space. Intuitively, one would expect such a measure to possess a support, where by the support it is meant the smallest closed set of full measure. For example, let $\mu$ be a Lebesgue measure on a cube $[0,1]^{2}$ in a plane. Then the support of $\mu$ is the entire cube, because otherwise one would need some non-empty open set of a zero measure. However, it appears that measures not possessing a support do exist.

The purpose of this paper is to present the construction of the so-called Dieudonné measure - a Borel measure possessing no support, as well as giving a simplified, up to our best knowledge unknown so far, example. The measure possessing no support was found by Jean Dieudonné in 1939 (see [1]). It appears that his ingenious and enlightening example is not difficult to explain to a non-specialist. For his construction, Dieudonné used tools that were already known in the very beginning of the $\mathrm{XX}^{\text {th }}$ century, introduced by Gerhard Hessenberg in 1906 (see [2]). By now the notions given by Hessenberg have become classical tools in set theory. The Dieudonné discovery ignited several deep investigation topics that have resulted in completely new discoveries, not only in measure theory, but also in set theory. This example has contributed to the understanding of the concept of a measureable cardinal number given by Ulam in [3]. A great development of measure theory on abstract topological spaces was made since then by, among others, Alexandroff [4], Rohlin [5] and Marczewski [6-8]. The most notable development was the notion of $\tau$-additivity of measures and its detailed study in series of papers by Alexandroff [4], which are being considered as a milestone in the field of abstract measure theory. This seems to be widely inspired by the construction given by Dieudonné.

However, the construction may be found in a variety of places after Dieudonné's paper, as for example in [9, Example 7.1.3], the existing presentations are hard to follow for non-specialists in set theory or abstract analysis. Perhaps this is the reason why this beautiful construction is not as commonly known as it deserves to be. It was the author's aim to present a possibly self-contained proof, which would be widely accessible for non-specialists. We also present another example of a non-supported measure, which is based

${ }^{\star}$ Corresponding Author: Piotr A. Kozarzewski: Faculty of Mathematics, Informatics and Mechanics, University of Warsaw, ul. Banacha 2, 02-097, Warsaw, Poland, E-mail: piotr.kozarzewski@mimuw.edu.pl

Faculty of Cybernetics, Military University of Technology, ul. Gen. Witolda Urbanowicza 2, 00-908, Warsaw, Poland

Oopen Access. (c) Br-Nc-ND (C) 2018 Piotr A. Kozarzewski, published by De Gruyter. This work is licensed under the Creative Commons Attribution-NonCommercial-NoDerivs 4.0 License. 
on Dieudonné's ideas, but the new construction allows to simplify it. Contrary to the classical examples, the measure we present is not finite. On the other hand, the construction is essentially simpler. To our best knowledge, infinite measures constructed on ordinal numbers have not been considered to far. The analysis of supports of Borel measures is visible in recent papers dealing with DiPerna-Majda measures, which are modern tools in the Calculus of Variations [10-12], seem influential in PDEs [13, 14] and applied mathematics, like the analysis of microstructures [15] or fluid mechanics [16].

Especially in [10], dealing with DiPerna-Majda measures, one may observe that the problem of the existence of the support of a measure seems to be an important topic for formulating essential assumptions on the main theorem. It was puzzling to the author if a violation of this assumption could lead to a construction experiencing similar phenomena. Such consideration could possibly weaken the standard assumptions in the field. This led to the surprising idea of connecting Calculus of Variations - in fact very applicable for engineering - with very abstract measure theory. It appears that these theories meet when looking for conditions guaranteeing the existence of the support of a Borel measure.

The paper begins with recalling some introductory information about the notions we deal with. Throughout the process, we introduce measure-theoretic and set-theoretic notions and prove needed properties. In the last part of the paper, the desired example comes easily as a consequence of presented considerations, as well as the new simplification is given. Although the paper is intended to be self-contained, some technical details are left to the reader to figure out. Even then, we refer to some outer sources where the facts are directly established.

\section{Auxiliary measure- and set-theoretic definitions}

\subsection{Classical measure-theoretic notions}

We start by recalling some measure-theoretic definitions and begin studying measures possessing support in a wide class of spaces, containing most classical examples.

Definition 2.1 (Measure, see Definition 1.3.2 in [9]). Let $\Omega$ be any set and $\mathcal{F}$ - any $\sigma$-field of subsets of $\Omega$. By a measure on $\Omega$ (measuring elements of $\mathcal{F}$ ) we mean a function

$$
\mu: \mathcal{F} \rightarrow[0,+\infty]
$$

which vanishes on $\emptyset$ and for any countable family of subsets $\left\{A_{i}\right\}_{i \in \mathbb{N}}$ of $\mathcal{F}$ such that $i \neq j \Rightarrow A_{i} \cap A_{j}=\emptyset$, we have

$$
\mu\left(\bigcup_{i} A_{i}\right)=\sum_{i} \mu\left(A_{i}\right)
$$

Definition 2.2 (Support of a measure, just above Proposition 7.2.9 in [9]). By a support of measure $\mu$ we mean a closed set $C \stackrel{\text { def }}{=} \operatorname{supp} \mu$ such that

i) $\mu(\Omega \backslash C)=0$;

ii) if $C_{1}$ is closed and $\mu\left(\Omega \backslash C_{1}\right)=0$, then $C \subseteq C_{1}$.

We may thus interpret support as the smallest closed set which has a $\mu$-"almost empty" complement. In particular, if a support exists, it must be equal to the intersection of all closed sets whose complements have the measure 0 .

The question on the existence of a support of measure $\mu$ is very simple in typical situations - for example every Borel measure defined on a metric, separable space possesses a support. Even more generally, the following proposition holds.

Proposition 2.3. Let $\Omega$ be a topological space with a countable basis of topology and $\mu$ be a Borel measure. Then the support of the measure $\mu$ is well-defined. 
Proof. Let us take a family $\mathscr{C}$ of all closed subsets $C$ of $\Omega$ such that $\mu(\Omega \backslash C)=0$. We show that the following equality holds

$$
\mathfrak{S} \stackrel{\text { def }}{=} \bigcap_{C \in \mathscr{C}} C=\operatorname{supp} \mu \text {. }
$$

It is clear that $\mathfrak{S}$ is closed. It is also convenient, that if $C$ is closed and $\mu(\Omega \backslash C)=0$, then $\mathfrak{S} \subseteq C$, because $C \in \mathscr{C}$. From that point we know that it is the smallest closed set in the family $\mathscr{C} \cup\{\mathfrak{S}\}$.

It remains to check, however, that $\mu(\Omega \backslash \mathfrak{S})=0$, i.e. $\mathfrak{S} \in \mathscr{C}$. To that end, let us denote the countable basis of topology on $\Omega$ as $\mathscr{U} \stackrel{\text { def }}{=}\left\{U_{i}\right\}_{i \in \mathbb{N}}$. Notice then, that for any closed $C \subseteq \Omega$ the set $\Omega \backslash C$ is open and thus it may be written as a sum of some sets $U_{i} \in \mathscr{U}$. For every closed $C \subseteq \Omega$ let us define

$$
I(C) \stackrel{\text { def }}{=}\left\{i \in \mathbb{N}: U_{i} \subseteq \Omega \backslash C\right\} .
$$

In this way, for every closed $C \subseteq \Omega$ we have

$$
\Omega \backslash C=\bigcup_{i \in I(C)} U_{i} .
$$

Notice that for every $C \in \mathscr{C}$ and every $i \in I(C)$ we have $\mu\left(U_{i}\right)=0$. Hence we have

$$
\Omega \backslash \mathfrak{S}=\bigcup_{C \in \mathscr{C}} \Omega \backslash C=\bigcup_{C \in \mathscr{C}} \bigcup_{i \in I(C)} U_{i}=\bigcup_{\{i \in \mathbb{N}:} \bigcup_{C \in \mathscr{C}} U_{i \in I(C)\}} .
$$

The set $\left\{i \in \mathbb{N}:{ }_{C \in \mathscr{C}} i \in I(C)\right\}$ is of course countable, because it is a subset of $\mathbb{N}$. Thus $\Omega \backslash \mathfrak{S}$ is a countable union of sets of measure 0 and thus it is of measure 0 .

Remark 2.4. Note that any metric separable space has a countable basis of topology. For the proof it is enough to take balls centred in the dense countable subset with rational radii. On the other hand, any space with a countable basis of topology is separable - for the proof one takes a single point from every open set of the countable basis.

Remark 2.5. The proposition above, shown for example in [9, Proposition 7.2.2 (iv)], is a special case of some deeper theorems, such as [9, Proposition 7.2.9].

\subsection{Ordinal numbers}

We move to recalling the definition of ordinal numbers and some basic facts about them. We will apply them to create a certain "very inseparable" topological space where our desired non-supported measure will lie. Let us also recall that by a well-ordered set we mean a set with such a relation of ordering, where every subset possesses its smallest element.

We begin with defining ordinal numbers in a non-standard, but very compact manner. The interested reader is welcome to compare this notion with [17, Excersices 12.2-12.4].

Definition 2.6 (Ordinal numbers). We define ordinal numbers by the procedure of transfinite induction. Take

(i) $0 \stackrel{\text { def }}{=} \emptyset$;

(ii) for any ordinal number $\alpha$ we define $\alpha+1 \stackrel{\text { def }}{=} \alpha \cup\{\alpha\}$;

(iii) for any set of ordinals $\left\{\alpha_{t}\right\}_{t \in T}$, where $T$ is any set of indices, we define the ordinal number $\sup \left\{\alpha_{t}\right\}_{t \in T} \stackrel{\text { def }}{=}$ $\bigcup_{t \in T} \alpha_{t}$.

Remark 2.7. It is worth noting that simultaneously we have $\alpha \in \alpha+1$ and $\alpha \subset \alpha+1$.

Example 2.8. Having $0=\emptyset$ already defined, note that step (ii) gives us

$$
1=\emptyset \cup\{\emptyset\}=\{\emptyset\}=\{0\} .
$$


We then have

$$
2=1 \cup\{1\}=\{\emptyset\} \cup\{\{\emptyset\}\}=\{\emptyset,\{\emptyset\}\}=\{0,1\} .
$$

Following this procedure we obtain the set of natural numbers (understood as the set of all finite ordinal numbers), having in mind that for any natural number $n$ we have

$$
n+1=\{0,1,2, \ldots, n\} .
$$

Note now that this is the moment when, for every already defined ordinal number $n$, the ordinal number $n+1$ is also defined and thus we may not use step (ii) for defining new ordinal numbers. Applying step (iii) to the set of natural numbers defined in this way we get the first infinite ordinal number

$$
\omega_{0}=\bigcup_{n \in \mathbb{N}} n=\bigcup_{n \in \mathbb{N}}\{0,1,2, \ldots, n-1\}=\{0,1,2, \ldots\},
$$

which is a supremum of all natural numbers. After that, step (ii) defines the ordinal number $\omega_{0}+1=$ $\left\{0,1,2, \ldots, \omega_{0}\right\}$. Continuing analogously, we will get the ordinal number $\omega_{0}+n=\left\{0,1, \ldots, \omega_{0}, \omega_{0}+\right.$ $\left.1, \ldots \omega_{0}+(n-1)\right\}$ and then applying step (iii) one gets $\omega_{0}+\omega_{0}=\left\{0,1, \ldots, \omega_{0}, \omega_{0}+1, \ldots\right\}$. We will denote this number as $2 \times \omega_{0}$.

Proceeding further, we will then obtain $2 \times \omega_{0}+n=\left\{0,1, \ldots \omega_{0}, \omega_{0}+1, \ldots, 2 \times \omega_{0}, 2 \times \omega_{0}+1, \ldots, \omega_{0}+\right.$ $(n-1)\}$ and applying step (iii) again, $3 \times \omega_{0}$. Similarly, we will obtain ordinal numbers $4 \times \omega_{0}, 5 \times \omega_{0}$ and, as the supremum of numbers $n \times \omega_{0}$, arrive at $\omega_{0} \times \omega_{0}$.

From the definition it follows that every ordinal number $\alpha$ is defined as a set consisting of all ordinal numbers previously defined. Furthermore, for any two ordinal numbers $\alpha \neq \beta$ we confirm that exactly one of the rela-

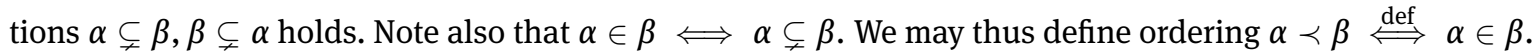
Any ordinal number $\gamma$ is a well-ordered set with such ordering, which seems visible, but is quite demanding from the technical side. Also, for any well-ordered set $T$ (with order $<$ ) there exists precisely one ordinal number $\vartheta$ (with order $\prec$ ) and bijection $\varphi: T \rightarrow \vartheta$ such that $t_{1}<t_{2} \Longleftrightarrow \varphi\left(t_{1}\right) \prec \varphi\left(t_{2}\right)$. The interested reader could find many details for example in [17, Chapter 12]. In particular, a precise proof of the well-ordering of ordinal numbers is given in [17, Theorem 12.15].

Definition 2.9 (Successor, compare to 12.2 in [17]). The number $\alpha$ is called a successor (of $\beta$ ), if there exists an ordinal number $\beta$ such that $\beta+1=\alpha$.

Successors are precisely the numbers which (as sets) possess the largest element. Other numbers, i. e. those not possessing its biggest element, are called limit numbers.

Example 2.10. Every non-zero natural number $n$ is, of course, a successor because $n=(n-1)+1$. On the other hand, $\omega_{0}$ is a limit number. For every $n \neq 0$, however, $\omega_{0}+n$ is a successor and $2 \times \omega_{0}$ is again a limit number.

We continue with defining certain symbols. By $\omega_{0}$ we will mean the first non-empty limit ordinal number, as in Example 2.8.

By $\omega_{1}$ we will mean the first ordinal number which is non-bijective (as a set) with $\omega_{0}$.

Then by the transfinite induction let us set $\omega_{\alpha}$ to be the first ordinal number which is non-bijective with $\omega_{\beta}$ for every $\beta<\alpha$.

It is probably worth explaining how the transfinite induction works in the particular situation of defining numbers $\omega_{\alpha}$. The induction is made with respect to the indices. The first point is then to define number $\omega_{0}$ and this is done with natural numbers, i.e. the number $\omega_{0}$ coincides with the first infinite ordinal number. Then, having defined $\omega_{\beta}$ we may define a number $\omega_{\beta+1}$ as the first ordinal number non-bijective with $\omega_{\beta}$. What is left to explain is the limit step. Having defined $\omega_{\beta}$ for every number $\beta<\alpha$ we may define number $\omega_{\alpha}$ as the first ordinal number which is non-bijective with any of numbers $\omega_{\beta}$. 
It is not visible at the first glance that the procedure of defining ordinal numbers $\omega_{\alpha}$ does not stop, i.e. one may wonder if there exists some 'untouchable' ordinal number $\delta$ such that there exists no $\omega_{\delta}$. The following observation shows that this is not the case.

Proposition 2.11 (Existence of ordinal numbers, 12.29 in [17]). For any ordinal number $\alpha$ there exists the ordinal number $\omega_{\alpha}$.

Proof. Zermelo's Theorem [17, Theorem 8.9] states that any set may be well-ordered. Let us also recall Cantor's Theorem [17, Theorem 2.21], stating that the set $P(T)$ of all subsets of a set $T$ has more elements than the set $T$ itself (in the sense that there exists no bijection between these sets, while obviously there exists a one-to-one function from $T$ to $P(T)$ - namely $t \mapsto\{t\}$ ).

Suppose then that there exists an ordinal number $\alpha$ such that $\omega_{\alpha}$ does not exist. From the procedure of the transfinite induction it follows that for any $\beta>\alpha$ the number $\omega_{\beta}$ also does not exist.

We may assume then that every ordinal number $\gamma$, such that there exists $\omega_{\gamma}$, is bounded from above by $\alpha$, hence these numbers $\gamma$ form an ordinal number which is a subset of $\alpha$. Let us call this number $\Gamma$. Note that $\Gamma \notin \Gamma$, so specifically there exists no $\omega_{\Gamma}$. Take $\Gamma^{\prime}=\sup _{\gamma \in \Gamma} \omega_{\gamma}$. Then the set $P\left(\Gamma^{\prime}\right)$ has more elements than any of the sets $\omega_{\gamma}$. Via Zermelo's Theorem this set may be well-ordered, so there exists an ordinal number bijective to $P\left(\Gamma^{\prime}\right)$. It follows that there exists an ordinal number which has more elements than any of the numbers $\omega_{\gamma}$ for $\gamma \in \Gamma$.

We will show that there exists a number $\omega_{\Gamma}$, which provides a contradiction. Indeed, we have shown that there exists an ordinal number (let's call it $\vartheta$ ) which has more elements than $\omega_{\gamma}$ for any $\gamma \in \Gamma$. Note now that $\vartheta \in \vartheta+1$. The set of those elements of $\vartheta+1$, which have more elements than $\omega_{\gamma}$ for any $\gamma \in \Gamma$ is thus non-empty and hence possesses its smallest element. From the very definition, this element is precisely $\omega_{\Gamma}$, thus $\omega_{\Gamma}$ exists - a contradiction.

Definition 2.12 (Cardinal numbers, 12.26 in [17]). The class of such ordinals $\alpha$ that there exists an ordinal number $\beta$ such that $\alpha=\omega_{\beta}$ will be called the class of cardinal numbers. We will say that a set $T$ is of cardinality $\kappa$ whenever $\kappa$ is such a cardinal number that $T$ is bijective with $\kappa$ and denote $|T|=\kappa$.

Note that any cardinal number is a limit ordinal number. This is because of the bijection between numbers $\alpha$ and $\alpha+1$, which holds whenever $\alpha$ is infinite.

Having the notion of the cardinality of a set, it is necessary to mention the classical and deep Hessenberg Theorem, which is attributed to the German mathematician Gerhard Hessenberg and dates back to 1906. The first proof of the result was presented by Hessenberg in [2]. To find a proof in English in any outer source, the author recommends [17, Lemma 9.15]. We will present the proof only in the case of a countable set $T$, because this is the only case we will use and the proof is significantly simpler.

Theorem 2.13 (Hessenberg in [2], 1906). Let $T$ be an infinite set. Then $|T|=|T \times T|$.

Proof - only in case of $T$ countable. First we prove that whenever sets $T, T^{\prime}$ satisfy $|T|=\left|T^{\prime}\right|$, then also $|T \times T|=$ $\left|T^{\prime} \times T^{\prime}\right|$. For that it is enough to check that whenever $\phi: T \rightarrow T^{\prime}$ is a bijection, so is $\tilde{\phi}: T \times T \rightarrow T^{\prime} \times T^{\prime}$ defined via $\tilde{\phi}\left[\left(t_{1}, t_{2}\right)\right]=\left(\phi\left(t_{1}\right), \phi\left(t_{2}\right)\right)$, which is standard.

It follows that it is sufficient to check that $|\mathbb{N}|=|\mathbb{N} \times \mathbb{N}|$, as by the assumption there exists a bijection $\phi: \mathbb{N} \rightarrow T$. This is done by a standard diagonal procedure, which is presented below for completeness.

Let us define the ordering $\prec$ on the set $\mathbb{N} \times \mathbb{N}$ as follows. We say $(n, m) \prec\left(n^{\prime}, m^{\prime}\right)$ whenever either $n+m<$ $n^{\prime}+m^{\prime}$ or $n+m=n^{\prime}+m^{\prime}$ and $n<n^{\prime}$. It is obvious that $\prec$ is a well-ordering of $\mathbb{N} \times \mathbb{N}$ and thus, there exists an ordinal number $\alpha$ and bijection $\psi: \mathbb{N} \times \mathbb{N} \rightarrow \alpha$ such that $(n, m) \prec\left(n^{\prime}, m^{\prime}\right) \Longleftrightarrow \psi[(n, m)]<\psi\left[\left(n^{\prime}, m^{\prime}\right)\right]$. Of course the set $\mathbb{N} \times \mathbb{N}$ is infinite, nevertheless for any pair $(n, m)$ there exist less than $\frac{1}{2}(n+m+1)^{2}$ (so finitely many) pairs $\left(n^{\prime}, m^{\prime}\right)$ such that $\left(n^{\prime}, m^{\prime}\right) \prec(n, m)$. Note that if $\omega_{0} \in \alpha$, then there exist infinitely many $\beta \in \alpha=\psi[\mathbb{N} \times \mathbb{N}]$ such that $\beta<\omega_{0}$. Hence $\omega_{0} \notin \psi[\mathbb{N} \times \mathbb{N}]$ and $\alpha$ is either finite or equal to $\omega_{0}$. As the first option is not the case, we see that $\mathbb{N} \times \mathbb{N}$ is bijective with $\omega_{0}$, which completes the proof of $|\mathbb{N}|=|\mathbb{N} \times \mathbb{N}|$. 
Remark 2.14. In particular from the Hessenberg Theorem it is simple to deduce, that whenever sets $T_{i}$ are countable, then $\bigcup_{i \in \mathbb{N}} T_{i}$ also is. Hence, $\omega_{1}$ is not a supremum of any countable family of its elements. We will use this fact in the sequel.

Let us skip to the ordinal topology, that is to the way we define the topology on ordinal sets. For that purpose we reformulate [9, Example 6.1.21] to a form more accurate for our purposes.

Definition 2.15 (Ordinal topology, compare to 12.24 in [17]). We say that subset $C$ of the ordinal $\alpha$ is closed in $\alpha$, if for any set $T$ and the choice of elements $c_{t} \in C$, where $t \in T$, the ordinal number $c$ defined via

$$
c \stackrel{\text { def }}{=} \bigcup_{t \in T} c_{t}
$$

belongs to $C$, whenever it belongs to $\alpha$.

Example 2.16. It follows straightforwardly from the definition of ordinal topology, that for any $\gamma<\alpha$ the set $C_{\gamma}=\{\beta \in \alpha: \beta>\gamma\}$ is closed in $\alpha$. It is also unbounded in the following sense: there is no such $\delta<\alpha$ (or equivalently $\delta \in \alpha$ ) such that $\beta \in C_{\gamma} \Rightarrow \beta \leq \delta$. In the sequel we will often refer to these two properties, namely to closeness and unboundedness. In the literature, closed and unbounded sets are often called clubs.

Definition 2.17 (Bounded and unbounded sets). Let $\alpha$ be an ordinal number. We say that the set $T \subset \alpha$ is bounded in $\alpha$, whenever there exists $\tau \in \alpha$ such that for each $t \in T$ the inequality $t<\tau$ holds.

Remark 2.18. Notice that for any limit number $\alpha$ and its unbounded subset $C$ (for example $C=\alpha$ ) the sum of the elements of $C$ is precisely $\alpha$ and thus does not belong to $\alpha$ as its element. This is the reason for the "whenever it belongs to $\alpha$ " condition in the definition of ordinal topology and actually the only case when it is used.

Remark 2.19. Such defined topology is somehow comparable to the typical "trace topology" of a subspace of a certain topological space. Note, however, that the interval $[0,1)$ is not closed in $\mathbb{R}$ (here we consider $\mathbb{R}$ with standard metric topology), but closed in the trace topology inherited from the interval $(-1,1)$. The same way the set $\mathscr{M}$ of all countable ordinals is closed and unbounded in $\omega_{1}$ (because it actually coincides with $\omega_{1}$ ) but in $\omega_{1}+1$ it is neither closed nor unbounded (because its supremum $-\omega_{1}$ - belongs to $\omega_{1}+1$ and does not belong to $\mathscr{M}$ ).

The ordinal $\omega_{0}$ can be homeomorphically embedded into [0,1] via $0 \mapsto 0$ and for $n \geq 1 n \mapsto 1-\frac{1}{n+1}$. This homeomorphism (onto the image) preserves order. It is worth observing that every point in $\omega_{0}$ is open, so we experience no problems with continuity. Similarly, one defines a map $\omega_{0}+1=\omega_{0} \cup\left\{\omega_{0}\right\} \rightarrow[0,1]$ via $n \mapsto 1-\frac{1}{n+1}, \omega_{0} \mapsto 1$. Intuitively, such defined function is continuous because the supremum of $n$ 's is mapped into the supremum of $\left(1-\frac{1}{n+1}\right)$ 's.

\subsection{Further set-theoretical tools}

The next proposition establishes an important feature of clubs, that is closed and unbounded sets. It will be crucial for the construction of the desired measure.

Proposition 2.20 (Compare to Lemma 3.4 in [18]). Let $\mathscr{C}$ be a countable family whose elements are closed and unbounded sets in $\omega_{1}$. Then the set $\bigcap_{C \in \mathscr{C}} C$ is closed and unbounded in $\omega_{1}$.

Proof. As the intersection of closed sets is always closed, it is sufficient to prove the unboundedness. Take then any $\gamma<\omega_{1}$. Let us also enumerate sets $C$ by natural numbers, so that $\mathscr{C}=\left\{C_{i}\right\}_{i \in \mathbb{N}}$. We will construct by induction a particular sequence $\left\{\xi_{i}\right\}_{i \in \mathbb{N}}$ of ordinals smaller then $\omega_{1}$. Let $\xi_{1}=\gamma$. Assuming now that for a certain $i$ that $\xi_{i}$ is well defined, let us define ordinal numbers $\vartheta_{i, j}$ for any $j \in \mathbb{N}$. For that purpose we take any 
$\vartheta_{i, j} \in C_{j}$ such that $\vartheta_{i, j}>\xi_{i}$. It is possible for every $j$, as every $C_{j}$ is unbounded in $\omega_{1}$. Let

$$
\xi_{i+1}=\sup _{j \in \mathbb{N}} \vartheta_{i, j}
$$

Now, by Remark 2.14, $\xi_{i+1} \in \omega_{1}$ because it is a supremum of a countable family of elements of $\omega_{1}$. Therefore, the sequence $\left\{\xi_{i}\right\}_{i \in \mathbb{N}}$ is properly defined.

Let us take $\delta=\sup \xi_{i}$ and note again that $\delta \in \omega_{1}$. Observe that for any fixed $j$ one has $\xi_{i} \prec \vartheta_{i, j} \prec \zeta_{i+1}$, and hence $\delta$ is also a supremum of elements of $C_{j}$. As a result, and from the closeness of $C_{j}$, we obtain that $\delta \in C_{j}$ for every $j$. Thus $\delta$ belongs to the intersection of $C_{j}$. In particular, the intersection of $C_{j}^{\prime} s$ possesses an element bigger than $\gamma$. As $\gamma$ was arbitrary, the unboundedness is established.

Now we are ready to define the $\sigma$-field of measurable sets for the Dieudonné measure. Let us take

$$
\begin{array}{r}
\mathscr{B} \stackrel{\text { def }}{=}\left\{A \subseteq \omega_{1} \mid \text { there exists some closed and unbounded set } C \text { in } \omega_{1}\right. \text { such that either } \\
\left.C \subseteq \subseteq \text { or } C \subseteq A^{\complement}\right\} .
\end{array}
$$

We will now make some observations regarding the family $\mathscr{B}$, using Remark 2.14 and Proposition 2.20.

Corollary 2.21. The following conditions hold

i) $\mathscr{B}$ is a $\sigma-$ field. Furthermore, every Borel set belongs to $\mathscr{B}$.

ii) Sets

$\mathscr{B}_{1} \stackrel{\text { def }}{=}\left\{A \subseteq \omega_{1} \mid\right.$ there exists some closed and unbounded set $C$ in $\omega_{1}$ such that $\left.C \subseteq A\right\}$ and

$\mathscr{B}_{2} \stackrel{\text { def }}{=}\left\{A \subseteq \omega_{1} \mid\right.$ there exists some closed and unbounded set $C$ in $\omega_{1}$ such that $\left.C \subseteq A^{\complement}\right\}$

are disjoint.

iii) If for every $i \in \mathbb{N}$ the set $A_{i} \in \mathscr{B}_{2}$, then $\bigcup_{i \in \mathbb{N}} A_{i} \in \mathscr{B}_{2}$.

Proof. First, let us note that whenever $C_{1}, C_{2}$ are closed and unbounded, the set $C_{1} \cap C_{2}$ is also closed and unbounded, so in particular is non-empty. On the other hand, if $C_{1} \subseteq A, C_{2} \subseteq A^{\complement}$, then $C_{1} \cap C_{2}$ is empty. Thus, $A$ is an element of at most one of sets $\mathscr{B}_{1}, \mathscr{B}_{2}$. This finishes the proof of part ii).

Obviously $\emptyset \subseteq \omega_{1}$ and $\omega_{1}$ is closed and unbounded, so taking $C=\omega_{1}$ in the definition of $\mathscr{B}$ shows that both $\emptyset$ and $\omega_{1}$ of them belong to $\mathscr{B}$.

From Proposition 2.20 it readily follows, that whenever for every $i \in \mathbb{N}$ one has $C_{i} \subseteq A_{i}$, then $\cap C_{i}$ is closed and unbounded and $\cap C_{i} \subseteq \cap A_{i}$. In addition, whenever $A_{1}$ is such that $C_{1} \subseteq A_{1}^{\complement}$ and $A_{i} \in \mathscr{B}$ for any $i$, then $C_{1} \subseteq A_{1}^{\complement} \subseteq\left(\cap A_{i}\right)^{\complement}$. We have shown then that a countable intersection of sets from $\mathscr{B}$ belongs to $\mathscr{B}$.

Finally - whenever $A$ is an element of particular $\mathscr{B}_{i}$, the set $A^{\complement}$ is an element of the other one. In fact this is straightforward because $\left(A^{\complement}\right)^{\complement}=A$. We have established that the complements of sets from $\mathscr{B}$ belong to $\mathscr{B}$ and thus, in view of the two previous paragraphs, proved that $\mathscr{B}$ is a $\sigma$-field.

It is left for us to observe that any closed set $K$ is either unbounded (and thus belong to $\mathscr{B}$, because $K \subseteq K$ ) or bounded by some $\gamma<\omega_{1}$. In the latter case note that the set $C_{\gamma}=\{\beta \in \alpha: \beta>\gamma\}$ is closed and unbounded, so there exists a closed and unbounded set $C$ such that $C \subseteq K^{\complement}$. Since then every closed set belongs to $\mathscr{B}$. Knowing that $\mathscr{B}$ is a $\sigma$-field, we conclude that any Borel set belongs to $\mathscr{B}$ and establish point i).

Point iii) is already established throughout the proof of ii), but it seems necessary for the sequel and thus we will formulate and prove it separately. Take closed and unbounded sets $C_{i}$ such that $C_{i} \subseteq A_{i}^{\complement}$. From Proposition 2.20 it follows that $\bigcap C_{i}$ is closed and unbounded. Thus one gets $\cap C_{i} \subseteq \cap A_{i}^{\complement}=\left(\bigcup A_{i}\right)^{\complement}$.

Remark 2.22. It is worth to stress, that whenever $C$ is a closed and unbounded set, it obviously belongs to $\mathscr{B} 1$. 


\section{An example of a Borel measure with no support}

Let us now construct the non-supported measure on the topological space $\omega_{1}$. For any $S \subseteq \omega_{1}$ such that $S \in \mathscr{B}$ we define measure

$$
\mathscr{D}(S)= \begin{cases}1 & \text { whenever } S \in \mathscr{B}_{1} \\ 0 & \text { whenever } S \in \mathscr{B}_{2} .\end{cases}
$$

Proposition 3.1. With such defined $\mathscr{D}$ we have

i) $\mathscr{D}$ is a Borel measure on $\Omega$;

ii) for every element $\beta \in \Omega$ there exists a closed set $C_{\beta}$ of measure 1 such that $\beta \notin C_{\beta}$. In particular, the intersection of all closed sets with complements of measure 0 is empty.

Proof.

i) First let us check that the measure is properly defined. As sets $\mathscr{B}_{1}$ and $\mathscr{B}_{2}$ are disjoint, every set has been assigned with precisely one measure. The measure of empty set is 0 , so we need to check additivity on at most countable families of disjoint sets. As Corollary 2.21 part iii) shows, a countable sum of sets with a measure 0 has a measure 0 . On the other hand, from Proposition 2.20 we see that any at most countable family of sets of measure 1 has a non-empty intersection (even of measure 1 ), hence the sets are not disjoint. It easily follows then, that $\mathscr{D}$ is a well-defined measure. From 2.21 part i) it follows that this measure is Borel.

ii) Indeed, for any $\beta$ take $C_{\beta}=\{\gamma \in \Omega \mid \gamma>\beta\}$ as in Example 2.16. Every set $C_{\beta}$ is closed and unbounded, thus of measure 1. Its complement is hence of measure 0 . As $\beta \notin C_{\beta}$, in particular $\beta \notin \bigcap_{\alpha \in \Omega_{1}} C_{\alpha}$. Arbitrariness of $\beta$ shows that $\bigcap_{\alpha \in \Omega_{1}} C_{\alpha}=\emptyset$.

The Dieudonné example is in fact an inspiration for plenty of other, just slightly more involving, examples. Let us mention that a technical modification of Proposition 2.20 reads as follows.

Proposition 3.2. Let $\alpha$ be any non-zero, non-limit ordinal number and $\mathscr{C}-$ a countable family whose elements are closed and unbounded sets in $\omega_{\alpha}$. Then the set $\bigcap_{C \in \mathscr{C}} C$ is closed and unbounded in $\omega_{\alpha}$.

This statement may still be improved by the use of the notion of cofinality, however we do not intend to introduce it. Proposition 3.2 shows in fact, that whenever $\alpha$ is a non-limit ordinal number, a variant of Dieudonné measure may by found on $\omega_{\alpha}$. Namely, we define

$$
\begin{gathered}
\mathscr{B}_{1} \stackrel{\text { def }}{=}\left\{A \subseteq \omega_{\alpha} \mid \text { there exists some closed and unbounded set } C \text { in } \omega_{\alpha} \text { such that } C \subseteq A\right\} \quad \text { and } \\
\mathscr{B}_{2} \stackrel{\text { def }}{=}\left\{A \subseteq \omega_{\alpha} \mid \text { there exists some closed and unbounded set } C \text { in } \omega_{\alpha} \text { such that } C \subseteq A^{\complement}\right\}
\end{gathered}
$$

and set

$$
\mathscr{D}_{\alpha}(S)= \begin{cases}1 & \text { whenever } S \in \mathscr{B}_{1} ; \\ 0 & \text { whenever } S \in \mathscr{B}_{2} .\end{cases}
$$

In our closing remarks we intend to present one more, slightly easier example. To be more precise - one without any of the tools described in subsection 2.3. The notion of a measureable number, which was introduced by Ulam in [3], dealt with probabilistic measures. This is probably the reason why such examples as the one below were hardly ever considered. Despite making an effort, the author has not found such a simplification of Dieudonné construction in the existing literature. Let us define a simplified Dieudonné measure on $\omega_{1}$ via

$$
\mathscr{E} \mathscr{D}(S)= \begin{cases}+\infty & \text { if it unbounded in } \omega_{1} \\ 0 & \text { otherwise. }\end{cases}
$$


To check that $\mathscr{E} \mathscr{D}$ is a measure it is sufficient to note that set $S$ is bounded in $\omega_{1}$ if and only if it is at most countable. As we have proven, the countable union of countable sets stays countable, hence a countable sum of sets of $\mathscr{E} \mathscr{D}$-measure 0 is still of measure 0 . On the other hand, a support of this measure does not exist, as the identical reasoning has shown in case of $\mathscr{D}$-measure.

We have presented this example to show that the Dieudonné construction may be simplified. It is, however, vital to observe, that the $+\infty$ substitution in the definition is necessary. To see it, let us take two unbounded sets. Let $A$ be the set of all limit ordinal numbers in $\omega_{1}$ and $B$ be defined via $B \stackrel{\text { def }}{=}\{\alpha+1: \alpha \in A\}$. Of course $A$ consists only of limit numbers, while $B$ - only of successors. In particular, they are disjoint. It shows that having any real number $R$ instead of $+\infty$ in the definition of $\mathscr{E} \mathscr{D}$ would lead to an improper definition. Realizing this allows us once again appreciate the elegance of the construction given in [1].

Acknowledgement: I would like to thank Prof. Roman Pol for his wise recommendations on the existing literature in the field of measure theory. Also, I wish to thank Prof. Tadeusz Krauze, Mrs. Robin Krauze and Agnieszka Kałamajska for their helpful comments on the draft of this paper.

\section{References}

[1] Dieudonné J., Un exemple d'un espace normal non susceptible d'une structure uniforme d'espace complet, C. R. Acad. Sci. Paris, 1939, 209, 145-147

[2] Hessenberg G., Grundbegriffe der Mengenlehre: zweiter Bericht über das Unendliche in der Mathematik, Vandenhöck \& Ruprecht, Göttingen, 1906

[3] Ulam S., Zur Masstheorie in der allgemeinen Mengenlehre, Fund. Math., 1930, 16, 140-150

[4] Alexandroff A. D., Additive set functions in abstract spaces, Mat. Sbornik: 1940, 8(50), 307-348; 1941, 9(51), 563-628; 1943, 13(55), 169-238

[5] Rohlin V. A., On the fundamental ideas of measure theory, Mat. Sbornik, 1949, 25, 107-150

[6] Marczewski E., On compact measures, Fund. Math., 1953, 40, 113-124

[7] Marczewski E., Remarks on the convergence of measurable sets and measurable functions, Colloq. Math., 1955, 3, 118-124

[8] Marczewski E., Collected mathematical papers, PAN, Warszawa, 1996

[9] Bogachev V., Measure Theory, Volumes 1 and 2, Springer-Verlag Berlin Heidelberg, 2007

[10] Katamajska A., On Young measures controlling discontinuous functions, J. Conv. Anal., 2006, 13, 177-192

[11] Kałamajska A., Kružík M., Oscillations and concentrations in sequences of gradients, ESAIM: COCV, 2008, 14, 71-104

[12] Kružík M., Roubíček T., On the measures of DiPerna and Majda, Math. Bohem., 1997, 122, 383-399

[13] Alibert J., Bouchitté G., Non-uniform integrability and generalized Young measures, J. Convex Anal., 1997, 4, 125-145

[14] Chipot M., Kinderlehrer D., Equilibrium configurations of crystals, Arch. Rational. Mech. Anal., 1988, 103, 237-277

[15] Kružík M., Prohl A., Young measure approximation in micromagnetics, Numer. Math., 2001, 90, 291-307

[16] DiPerna R., Majda A., Oscillations and concentrations in weak solutions of the incompressible fluid equations, Comm. Math. Phys., 1987, 108, 667-689

[17] Moschovakis Y., Notes on Set Theory, Springer Science and Business Media, 2006

[18] Hrbacek K., Jech T., Introduction to Set Theory, Third Edition, Revised and Expanded, CRC Press, 1999 